\title{
Diabetes insipidus is an unfavorable prognostic factor for response to glucocorticoids in patients with autoimmune hypophysitis
}

\author{
Isabella Lupi', Mirco Cosottini' ${ }^{2}$, Patrizio Caturegli³, Luca Manetti ${ }^{1}$, Claudio Urbani $^{1}$, \\ Daniele Cappellani ${ }^{1}$, Ilaria Scattina', Enio Martino', Claudio Marcocci ${ }^{1}$ and \\ Fausto Bogazzi ${ }^{1}$ \\ ${ }^{1}$ Department of Clinical and Experimental Medicine, Section of Endocrinology, University of Pisa, Pisa, \\ Italy, ${ }^{2}$ Department of Translational Research and New Surgical and Medical Technologies, University of Pisa, \\ Pisa, Italy, and ${ }^{3}$ Division of Immunology, Department of Pathology, The Johns Hopkins School of Medicine, \\ Baltimore, Maryland, USA
}

\author{
Correspondence \\ should be addressed \\ to I Lupi \\ Email \\ isabellalupi@yahoo.it
}

\begin{abstract}
Introduction: Autoimmune hypophysitis $(\mathrm{AH})$ has a variable clinical presentation and natural history; likewise, its response to glucocorticoid therapy is often unpredictable.

Objective: To identify clinical and radiological findings associated with response to glucocorticoids.

Design and methods: 12 consecutive patients with $\mathrm{AH}$, evaluated from 2008 to 2016 . AH was the exclusion diagnosis after ruling out other pituitary masses and secondary causes of hypophysitis. Mean follow-up time was $30 \pm 27$ months (range 12-96 months).

Results: MRI identified two main patterns of presentation: global enlargement of the pituitary gland or panhypophysitis $(n=4, \mathrm{PH})$, and pituitary stalk abnormality only, or infundibulo-neuro-hypophysitis $(n=8, \mathrm{NH})$. Multiple tropin defects were more common in PH $(100 \%)$ than those in INH $(28 \% P=0.014)$, whereas diabetes insipidus was more common in INH $(100 \%)$ than that in $\mathrm{PH}(50 \% ; P=0.028)$. All $4 \mathrm{PH}$ and 4 out of 8 INH were treated with glucocorticoids. Pituitary volume significantly reduced in all $\mathrm{PH}$ patients $(P=0.012)$, defective anterior pituitary function recovered only in the two patients without diabetes insipidus (50\%) and panhypopituitarism persisted, along with diabetes insipidus, in the remaining 2 (50\%). In all INH patients, either treated or untreated, pituitary stalk diameter reduced $(P=0.008)$ but diabetes insipidus persisted in all.

Conclusions: Glucocorticoid therapy may improve anterior pituitary function in a subset of patients but has no effect on restoring posterior pituitary function. Diabetes insipidus appears as a negative prognostic factor for response to glucocorticoids.

\section{Introduction}

As autoimmune hypophysitis $(\mathrm{AH})$ is increasingly recognized in endocrinology practice, its diagnosis relies more frequently on clinical grounds, defined by presenting symptoms, MRI and laboratory tests, than on histological confirmation (1).

The clinical presentation of $\mathrm{AH}$ is variable according to the extension of inflammation: when the anterior lobe is
() 2017 European Society of Endocrinology Printed in Great Britain involved, as in adeno-hypophysitis or in panhypophysitis (PH), varying degrees of hypopituitarism and symptoms from mass compression, usually headache and/or visual disturbances, are expected. When the posterior lobe is involved along with the infundibulum, as in infundibuloneuro-hypophysitis (INH), diabetes insipidus is the most common symptom $(2,3)$. 
Treatment of AH comprises either medical therapy to reduce inflammation and the pituitary mass or pituitary surgery, especially when neurological symptoms are severe and medical therapy fails. Hormone replacement therapy does not affect the clinical course of $\mathrm{AH}$ and the inflammatory process; however, it can be started whenever hypopituitarism is present. Also, a watchful waiting could be an option when there is a high likelihood of self-resolution or when the risks of intervention or therapy may outweigh the benefits. Glucocorticoid therapy is proven to be beneficial in reducing the pituitary mass and related compression symptoms (4); its effectiveness on long-term restoration of pituitary function, however, is not clear and some authors suggest no therapy at all in specific cases of AH (5).

In the effort to further characterize clinical and radiological presentation and history of the disease, and to define whether glucocorticoid therapy has a role in improving the outcome, we analyzed 12 consecutive cases of $\mathrm{AH}$ that were referred to our Institution during the period 2008-2016.

\section{Patients and methods}

This was a retrospective cohort study, involving 12 consecutive patients with $\mathrm{AH}$, conducted in the timeframe 2008-2016 at the Endocrinology Unit of the University of Pisa. Although the study was retrospective, all patients were studied following the same work-up, and data were collected in a prospective manner.

Among patients with pituitary diseases who were evaluated at our Neuroendocrinology Service, about 1500/ year, and who performed hormonal examinations and pituitary MRI, we selected 25 individuals who presented with either anterior and/or posterior pituitary deficiency or compression symptoms (headache and visual disturbances) and sellar MRI features strongly suggestive of a pituitary inflammatory disease.

Twelve patients, 8 females (mean age $47 \pm 17$ years) and 4 males (mean age $55 \pm 13$ years), met the inclusion criteria for $\mathrm{AH}$ and were included in the study. As suggested in the algorithm for diagnosis and management of pituitary stalk lesions of the Mayo Clinic (6), the secondary causes of hypophysitis, such as infiltrative systemic diseases, neoplastic or congenital diseases were reasonably ruled out by performing additional examinations (chest
X-ray, or CT scan, abdominal ultrasound, serum or liquor HCG, serum ACE and long bones X-ray). Among the 13 patients excluded: 4 had pituitary hyperplasia, 3 Rathke cleft cyst, 2 Langerhans cell histiocytosis, 2 Erdheim-Chester disease, one Tolosa-Hunt disease and 1 metastatic breast cancer. In 10 cases, all the MRI findings, clinical and biochemical presentation and evidence at follow-up were suggestive of $\mathrm{AH}$. In 2 additional cases, $\mathrm{AH}$ was further confirmed by histopathology after a pituitary biopsy was performed. Since the diagnosis of $\mathrm{AH}$, patients were followed by regular outpatient visits, hormonal evaluation and pituitary MRI: every 6 months for the first 1 or 2 years after diagnosis, and then every 12 months. Mean follow-up time was $30 \pm 27$ months (range: 12-96 months).

A signed informed consent was obtained from all patients.

\section{Hormonal assessment}

All patients underwent basal endocrine evaluation of anterior pituitary function by measuring TSH, FT4, ACTH, cortisol, LH, FSH, testosterone/estradiol, prolactin and IGF-I.

To assess GH deficiency, a GHRH+arginine test was performed in all patients. GH cut-off values were applied according to $\mathrm{BMI}(7,8)$. To evaluate central hypoadrenalism, ACTH test (cosyntropin, $250 \mu \mathrm{g}$ i.m.) or CRF test was performed. A value of cortisol less than $181 \mu \mathrm{g} / \mathrm{dL}$ was indicative of low response (9). Hypogonadotropic hypogonadism was diagnosed with low levels of gonadotropins and testosterone in males, low levels of gonadotropins in post-menopausal females and low levels of gonadotropins and absence of menses in pre-menopausal women.

To assess posterior pituitary function, $24 \mathrm{~h}$ urinary volume and osmolality were measured. In patients with hypotonic polyuria (more than $50 \mathrm{~mL} / \mathrm{kg}$ of body weight $/ 24 \mathrm{~h}$ and osmolality less than $300 \mathrm{mosmol} / \mathrm{kg}$ ) (9), a water deprivation test was performed.

All patients were evaluated to exclude the presence of other organ-specific autoimmune diseases such as autoimmune thyroiditis, gastritis, Addison disease, celiac disease and diabetes mellitus type 1 by testing specific antibodies (Ab anti-thyroglobulin, thyroperoxidase, gastric parietal cells, 21-hydroxylase, transglutaminase and glutamic acid decarboxylase). 
In the effort to identify IgG4-related hypophysitis variant, fractionated serum IgG were measured in all patients.

\section{Detection of anterior pituitary antibodies by single indirect immunofluorescence}

The detection of serum antibodies against the anterior pituitary (Pit Abs) was performed by 'single indirect immunofluorescence' (the primary incubation only contained the serum of the participants diluted at 1:10) on a human pituitary gland substrate utilizing a recently published protocol (10). Sections were read independently by P C and G D D and considered positive for the presence of anterior Pit Abs when stained cells were present in at least 4 out of 20 microscopic fields.

\section{Pituitary MRI}

MRI examinations were performed using a 1.5T MR system. The MR protocol included SE T1-weighted sequences in the sagittal and coronal planes before and after administration of paramagnetic gadolinium (Gd) and FSE T2-weighted images in the coronal plane. Images were qualitatively evaluated by a single observer, and the following radiological features were collected: pituitary enlargement, adenohypophyseal signal hyperintensity on T2-weighted images, pituitary stalk thickening, the absence of neurohypophyseal hyperintensity, sphenoid sinus mucosal thickening and parasellar dural tail.

A quantitative analysis of MR findings was performed by measuring the signal intensity, the relative contrast enhancement, the volume of the pituitary gland and the thickness of the pituitary stalk. The signal intensity of the pituitary gland was measured on T1- weighted and T2-weighted images as the mean value obtained by placing two ROIs in the left and right portions of the adenohypophysis normalized on to the signal intensity of the white matter of the temporal lobe. The relative contrast enhancement was measured with an ROI method as the difference between the signal intensity obtained without and with contrast enhancement, normalized on the signal of the white matter of the temporal lobe. The pituitary volume was estimated using the ellipsoid formula (length $\times$ width $\times$ height $/ 2$ ) and expressed in $\mathrm{mm}^{3}$, while the pituitary stalk diameter was obtained by the mean value of the transverse and anteroposterior diameters measured at the point of maximum enlargement and expressed in $\mathrm{mm}$ (11). The signal intensity on T1-weighted and T2-weighted images the relative contrast enhancement on T1-weighted post-Gd, the pituitary volume and thickness of the pituitary stalk were measured at diagnosis and the end of follow-up. Ten healthy subjects matched for age and sex served as controls to compare T1and T2-weighted images.

\section{Treatment and follow-up}

Eight patients were treated with oral glucocorticoids (methylprednisolone $40 \mathrm{mg} /$ day gradually tapered in 6 months), none of them had conditions that contraindicated therapy. Blood pressure and glycemia were monitored periodically. All patients met the criteria to receive bisphosphonate to prevent bone loss (12) because of duration of therapy ( $>3$ months) and initial daily dose of glucocorticoids (>7.5 mg/day); in particular, 3 female patients in childbearing age, were also treated because of osteopenia (patient 8) and long-term hypogonadism (patients 4 and 5). Four patients refused glucocorticoid therapy. All the ten patients with diabetes insipidus were treated with desmopressin to control polyuria.

Recurrence of hypophysitis was defined as relapse of either hypopituitarism and/or compression symptoms and MRI evidence of an enlarging pituitary/stalk mass.

\section{Statistical analysis}

Quantitative data are expressed as mean \pm s.D. and qualitative data are expressed as a percentage.

Pituitary volume and stalk size at diagnosis and the end of follow-up were analyzed by two-tailed paired $T$-test for repeated measures. Difference in pituitary volume and stalk size among independent groups were analyzed by two-tailed unpaired T-test. A one-way ANOVA was conducted to compare the effect of pituitary inflammation on MRI T1 and T2 signal during active disease, at the end of follow-up and in controls. Bonferroni's post hoc test was conducted to measure the differences between the means. Qualitative data were analyzed by Fisher exact test.

$P$ value $<0.05$ was considered to be statistically significant. All analyses were performed using Prism, version 5.0 (GraphPad Software).

\section{Results}

\section{Symptoms of presentation in the whole series of patients}

Four out of 12 (33\%) patients presented with an intense headache, not remitting with high doses of nonsteroidal 

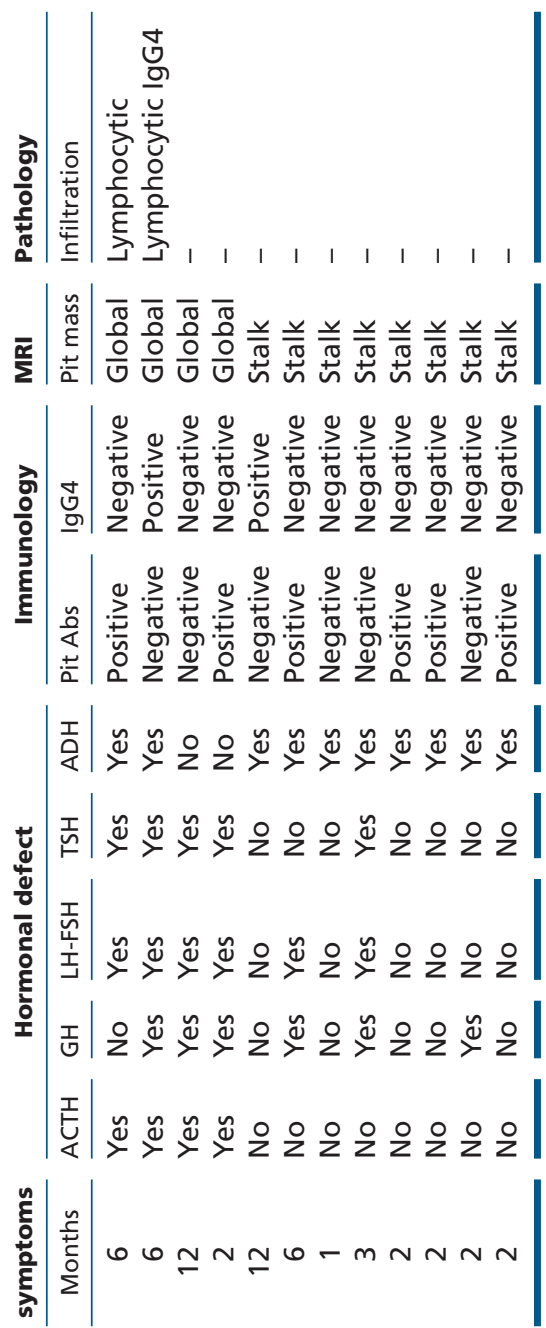

|

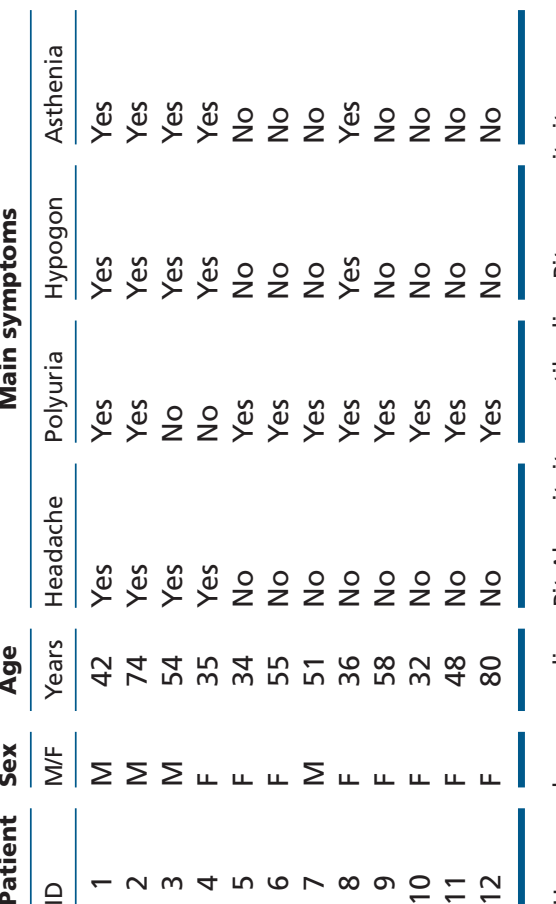

anti-inflammatory drugs. Five out of 12 (42\%) presented with symptoms due to hypopituitarism (mainly asthenia, loss of libido, amenorrhea), 10 out of 12 (83\%) with hypotonic polyuria and polydipsia. Duration of symptoms before diagnosis was variable, ranging from 1 to 12 months (mean $4.6 \pm 3.9$ months S.D., Table 1 ).

\section{MRI features}

At qualitative analysis of pituitary MRI, 4 out of 12 patients (33\%) had a global pituitary enlargement and stalk thickness, whereas 8 out of $12(66 \%)$ presented only with a visible abnormality of stalk imaging, including either a round-shape (37\%), a V-shape enlargement (37\%) or an elongated shape with increased brightness (25\%).

Based on MRI qualitative analysis, the cohort study was divided in panhypophysitis ( $\mathrm{PH})$ in the case of anterior pituitary and stalk enlargement ( $n=4$, Fig. 1B), and in INH in the case of stalk abnormality ( $n=8$, Fig. 1C).

In $\mathrm{PH}$ patients, the posterior bright spot was absent in all 4, mucosal thickening was evident in 3 out of 4 (75\%), and dural tail in 2 out of 4 (50\%), T1 and T2 hyperintensity of the pituitary gland was present in all 4 . Also, T2 dark signal intensity rim surrounding the pituitary gland was observed in 2 out of 4 (50\%) (13). All patients satisfied criteria of the proposed radiological score to differentiate hypophysistis from adenoma (score <0) (14).

In INH patients, the posterior bright spot was absent in all eight. A signal hyperintensity or mucosal thickening or dural tail was not found in any.

Mean pituitary volume at diagnosis was larger in $\mathrm{PH}$ $\left(1253 \pm 489 \mathrm{~mm}^{3}\right)$ than that in INH $(374 \pm 218, P=0.0013$, Fig. 2A), whereas mean stalk diameter did not differ $(\mathrm{PH}$ $3.42 \pm 0.99 \mathrm{~mm}$, INH $4.4 \pm 1.7 \mathrm{~mm}, P=0.31$, Fig. $2 \mathrm{~B})$. Mean T1 and mean T2 signals did not differ among PH, INH and controls. After gadolinium administration, however, contrast enhancement was significantly different among the 3 groups $(P<0.0001)$. In particular, mean T1 signal after gadolinium was significantly higher in $\mathrm{PH}$ than that in controls $(2.45 \pm 0.19$ and $1.71 \pm 0.15$ respectively, $P<0.001)$ and in PH than that in INH $(2.0 \pm 0.2, P<0.05)$, but did not differ between INH and controls (Fig. 2C).

\section{Hormonal status and immunological features}

Multiple tropin defects (2 or more) were found in all 4 (100\%) $\mathrm{PH}$ patients and 2 INH patients $(25 \%$, $P=0.014)$. Two out of $8(25 \%)$ INH patients had a single tropin defect, whereas a normal anterior pituitary 
A

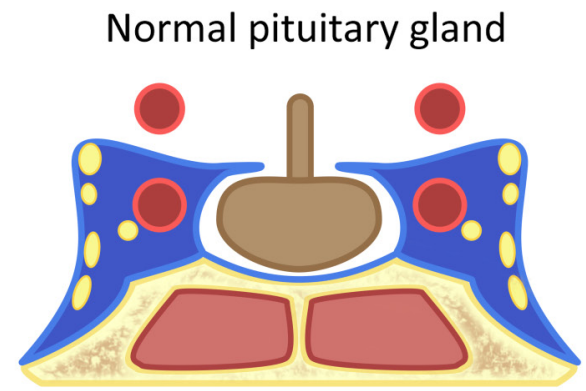

B

Pan-hypophysitis

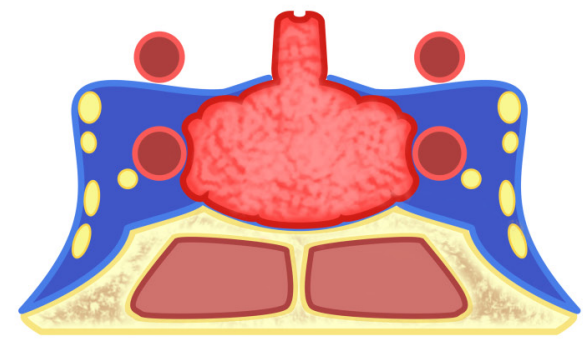

C Infundibulo-neuro-hypophysitis

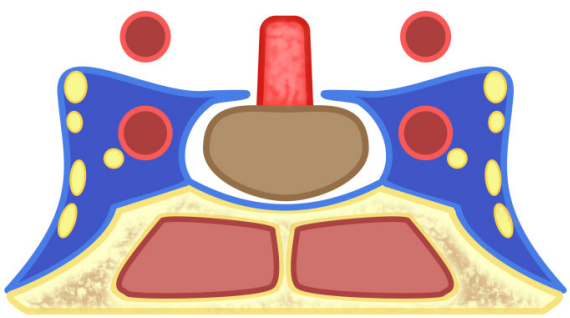

\section{Figure 1}

Imaging configurations of the pituitary gland lesions on MRI. Panel A: normal. Panel B: panhypophysitis. Panel C: infundibulo-neuro-hypophysits. A full colour version of this figure is available at http://dx.doi.org/10.1530/EJE-17-0123.

function was found in the remaining 4 (50\%). Mild hyperprolactinemia was present only in 3 out of 4 (75\%) $\mathrm{PH}$ patients. Central diabetes insipidus was found in 2 out of 4 (50\%) PH patients, and in all 8 INH patients (100\%) $(P=0.028$, Table 1$)$.

Pit Abs prevalence did not differ between groups: they were found in 2 out of $4(50 \%) \mathrm{PH}$ patients and in 4 out of $8(50 \%)$ INH patients.

Serum IgG4 ( $>140 \mathrm{ng} / \mathrm{dL}$ value) were found in 1 out of $4 \mathrm{PH}$ patients, in whom the diagnosis of IgG4related hypophysitis was confirmed by pathology after pituitary biopsy, and in 1 out of 8 INH patients. All the other organ-specific antibodies tested were absent in all 12 patients.
A

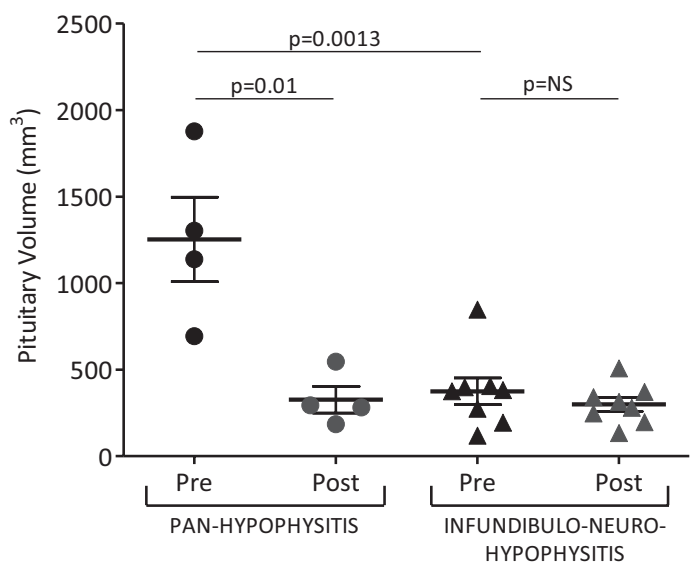

B
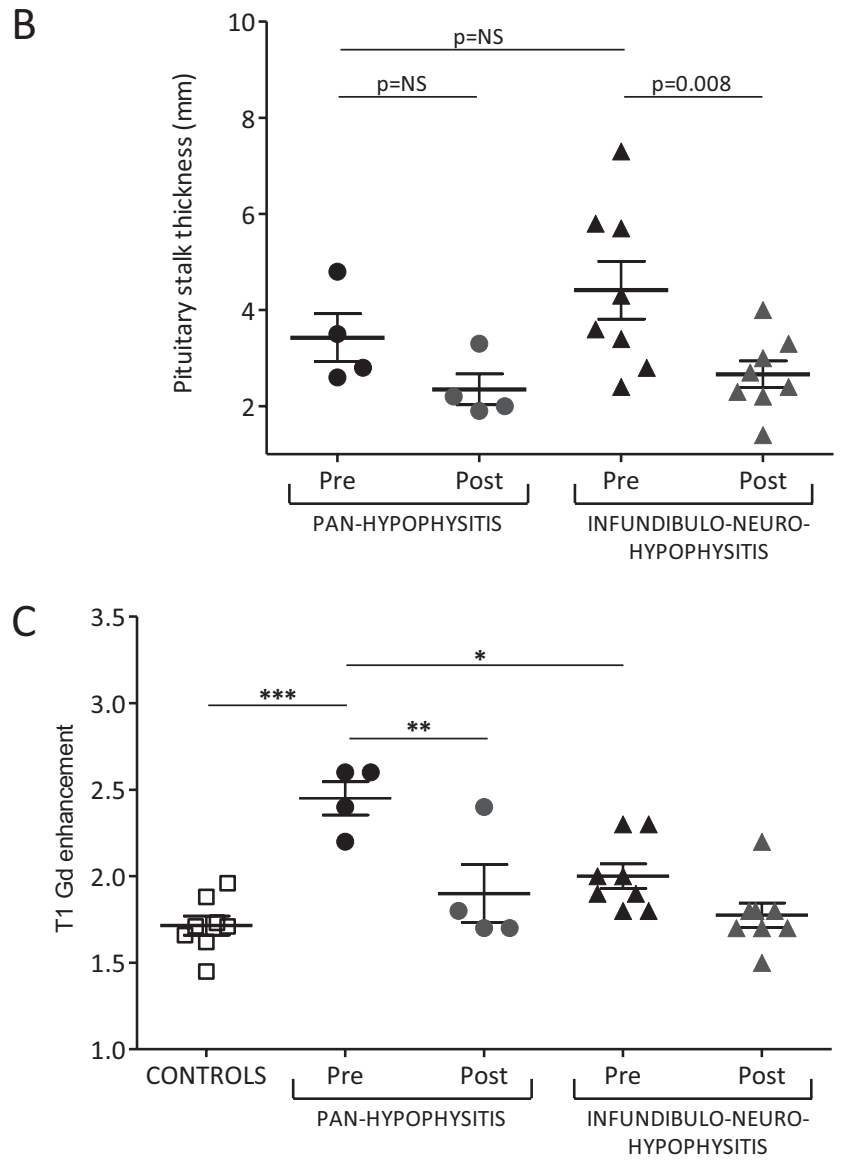

\section{Figure 2}

Pituitary MRI features. Panel A: pituitary volume $\left(\mathrm{mm}^{3}\right)$ at diagnosis and at the end of follow-up in panhypophysitis and in infundibulo-neuro-hypophysitis. Panel B: pituitary stalk diameter $(\mathrm{mm})$ at diagnosis and at the end of follow-up in panhypophysitis and in infundibulo-neuro-hypophysitis. Panel C: T1 enhancement after gadolinium at diagnosis and at the end of follow-up in panhypophysitis, in infundibulo-neurohypophysitis and in controls. ${ }^{*} P<0.05, * * P<0.01, * * * P<0.0001$ according to ANOVA followed by Bonferroni's post hoc test. 


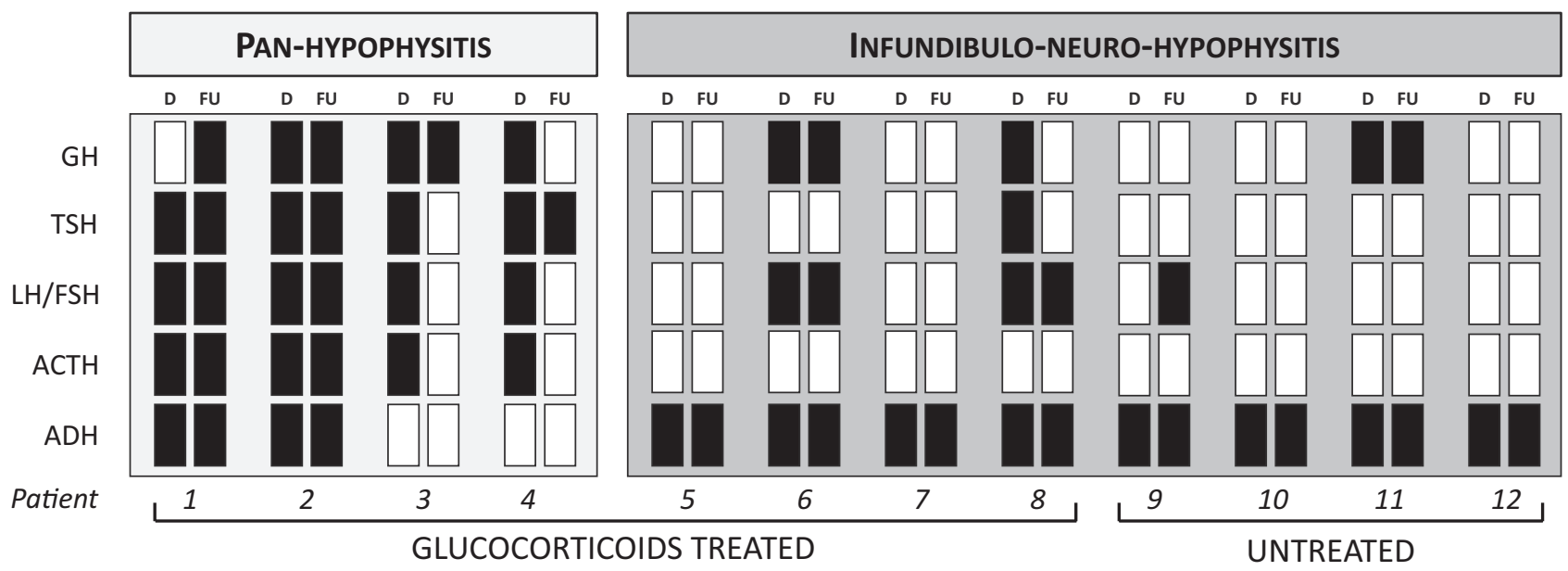

\section{Figure 3}

Hormonal defects of patients present at diagnosis and at the end of follow-up. White open boxes: Normal; Solid black boxes: Deficiency; D, diagnosis; FU, end of follow-up.

\section{Follow-up}

In $\mathrm{PH}$ treated patients $(n=4)$, headache disappeared within one week in all. Two patients (50\%) experienced a relapse of disease within 6 months, during low glucocorticoids dose $(10 \mathrm{mg} /$ day). In these two patients, the dose was increased at $40 \mathrm{mg} /$ day for 2 weeks, then gradually tapered. At the end of treatment (6 months after diagnosis), hypopituitarism improved from multiple to single tropin defect in 3 out of 4 patients (75\%). However, the improvement was only transient: after continuing follow-up in all four patients ( $54 \pm 36$ months), we observed that in 2 out of 4 , those who also experienced relapse of disease, new tropin defects appeared over time (Patient 1: GH, TSH and LH/ FSH, Patient 4: TSH as shown in Fig. 3). In the long term, relapse of hypophysitis was ever observed. Diabetes insipidus persisted in all patients.

In INH-treated patients $(n=4)$ hypopituitarism remained unchanged in $1(25 \%)$ and pituitary function improved from multiple to single tropin defect in 1 (25\%). In 2 out of 4 with normal function, no new tropin defects were observed. Diabetes insipidus remained unchanged in all 4 (Fig. 3).

In INH untreated patients $(n=4)$, anterior pituitary function worsened in 1 (25\%), whereas remained unchanged in 2 with normal function and in 1 with single tropin defect. Diabetes insipidus remained unchanged in all 4 (Fig. 3).

\section{Pituitary MRI features at the end of follow-up}

Mean MRI pituitary volume significantly decreased in $\mathrm{PH}$ $\left(326 \pm 154 \mathrm{~mm}^{3}(P=0.012)\right.$ but not in INH $\left(299 \pm 114 \mathrm{~mm}^{3}\right.$ $P=0.10$, Fig. 2A). When we further divided INH patients by treatment received (yes/no) and compared volume at diagnosis and the end of follow-up, we did not find any significant difference (untreated $P=0.18$, treated $P=0.19)$. Stalk diameter did not significantly decrease in $\mathrm{PH}(2.35 \pm 0.64 \mathrm{~mm}, P=0.13)$, whereas it did in $\mathrm{INH}$ (2.6 $\pm 0.78, P=0.008$, Fig. 2B).

Mean T1 and T2 signal did not significantly reduce when compared to active disease in both groups. After gadolinium administration, however, T1 signal was significantly lower in $\mathrm{PH}(1.9 \pm 0.33, P<0.05)$ but not in INH $(1.77 \pm 0.2, P=$ ns, Fig. $2 \mathrm{C})$.

\section{Discussion}

The main finding of the present study is that $\mathrm{PH}$ and INH differ not only in clinical presentation but also in response to glucocorticoids therapy.

Main clinical features of PH patients were a sudden occurrence of a headache, multiple hormone defects, evident in 100\% patients and diabetes insipidus, observed in $50 \%$. The inflammatory process was confirmed by the enlarged pituitary gland and stalk, which appeared T1 intense at MRI. Overall, this clinical and radiological 
presentation, further confirmed by pathology in $50 \%$ of the cases, made the diagnosis of $\mathrm{PH}$ rather simple.

Clinical presentation of INH was quite different: the main symptom was diabetes insipidus, present in $100 \%$ of the patients, whereas anterior pituitary defect, usually single, was found in a subset of patients (37\%). MRI demonstrated a pituitary stalk lesion, presenting as V-shape or round-shape thickness, or less frequently, as elongation and increased brightness. The pituitary T1 signal after gadolinium was overall higher than in controls, suggesting also an anterior pituitary involvement. It is worth noting that any stalk lesion might be the sign of neoplastic, infectious or inflammatory/infiltrative disease; unlike anterior pituitary masses, these lesions are rarely biopsied due to the critical location and crucial function of the stalk, therefore we can seldom rely on histological diagnosis. In this particular cohort of patients with INH, time and effort were taken first to exclude secondary causes of hypophysitis through additional biochemical and radiological examinations. Currently, MRI is the most powerful tool to differentiate $\mathrm{AH}$ from other pituitary masses, and a set of radiological signs has been identified to orient the clinician toward differential diagnosis with adenoma, the most common pituitary lesion (14). This radiological score, applied to $\mathrm{PH}$ patients, confirmed the diagnosis of AH. However, when inflammation is limited to pituitary stalk and infundibulum, as in INH, MRI cannot discriminate with certainty the nature of the lesion. In a retrospective study carried out on 152 patients over 20 years, Turcu et al. found that a V-shape or a roundshape of the stalk was the only feature most frequently associated with $\mathrm{AH}$ (6). Besides, in a recent study (15), it was reported that stalk lesions with a diameter greater than $9 \mathrm{~mm}$ were likely to be of neoplastic nature. The current series of patients satisfied either the morphological criteria or the stalk diameter criteria. It clearly appears, however, that radiological and clinical features, applied in conjunction with a prolonged follow-up are the key features to diagnose autoimmune INH.

It has been reported that in $\mathrm{AH}$, a complete, or partial hypopituitarism usually develops as consequence of pituitary infiltration. In our series, according to the extension of inflammation, we found a condition of multiple tropin defects in all patients with $\mathrm{PH}$ and a condition of partial hypopituitarism in only $37 \%$ of patients with INH. As regard of tropin involved, data from the literature indicate that the axis most commonly defective is the corticotroph, next are the thyrotroph and gonadotroph deficiencies. GH deficiency, in published patients, was not investigated in most of the cases
(2). In our series, we found a reverse picture: GH and gonadotropin defect in 50\%, TSH defect in $41 \%$, ACTH defect in 33\%. GH deficiency would have been unnoticed if a provocative test was not performed. It has been reported that GH or somatomammotropin can be a target autoantigen in the autoimmune attack $(16,17)$ thus, it is likely that a more extensive investigation of $\mathrm{GH}$ axis in $\mathrm{AH}$ patients will demonstrate an increased prevalence of GH deficiency.

The role of pituitary autoimmunity in clinical practice is still in question (18). Although a reliable IF method, based upon measurement of antibodies using a substrate of human pituitary, has been proposed (10) and utilized in this study, it still does not reach a sufficient sensitivity and specificity to diagnose AH with any certainty. Predictably, in our cohort, PitAbs were found in 50\% of cases, both in $\mathrm{PH}$ and in INH. Interpretation of these data suggests that, once again, after reasonable exclusion of secondary causes of hypophysitis, PitAbs positivity may reinforce the diagnosis of AH especially in the case of INH.

Glucocorticoids therapy has been reported to be effective in reducing pituitary mass, thus rapidly improving headache, in patients with $\mathrm{AH}$ (2). In our series of $\mathrm{PH}$ patients, a persistent improvement of anterior pituitary function was observed in 50\% although no effect was obtained on diabetes insipidus. In patients with $\mathrm{INH}$, at the end of follow-up, a significant pituitary stalk reduction either in treated or untreated was observed, suggesting that changes of pituitary stalk diameter may occur spontaneously. Moreover, similarly to what observed in $\mathrm{PH}$ patients, diabetes insipidus persisted in $100 \%$ of cases, and anterior pituitary function improved only partially.

Overall, although we cannot exclude that a spontaneous recovery might take place, our data suggest that patients with $\mathrm{PH}$ may benefit from glucocorticoids therapy mainly in the relief of compressive symptoms. Also, a recovery of anterior pituitary function may occur in PH patients without DI, whereas an involvement of the posterior pituitary seems to impair the response of adenohypophysis to glucocorticoids.

Patients with INH appear to be insensitive to glucocorticoids either in term of pituitary stalk mass and of the restoration of posterior pituitary function.

Why glucocorticoids work in some patients and not in others is a matter of discussion. We assume that an initial recovery of pituitary function might be due to the effect of glucocorticoids on reducing edema, although fibrotic changes may develop at later stages and affect pituitary function over time (19). When glucocorticoid therapy 
is started once fibrotic changes already occurred, any damage due to inflammation is not reversible. It is likely that the two PH patients with transient improvement, which also experienced a relapse of the disease, presented an aggressive type of hypophysitis that rapidly evolved toward fibrosis.

Limits of our study are the relatively small number of cases and its retrospective nature. Although reaching the statistical significance, due to the small sample size, the statistical analysis might have some limits and a larger series of patients would certainly be more representative. On the other hand, given the rarity of the disease, strength of this study is that patients were followed in a single center and data were collected following strict criteria and in a prospective manner.

In conclusion, we report the follow-up of a cohort of patients with $\mathrm{AH}$. We highlight the pitfalls in diagnosis, the different clinical and radiological presentation and response to glucocorticoids that we observed in $\mathrm{PH}$ compared to INH patients. Although glucocorticoids proved not successful in curing diabetes insipidus, treatment may still be proposed to $\mathrm{AH}$ patients with the goal to resolve compression symptoms and reduce the mass, when present. AH patients with diabetes insipidus should be considered for alternative therapies (20).

\section{Declaration of interest}

The authors declare that there is no conflict of interest that could be perceived as prejudicing the impartiality of this study.

\section{Funding}

This research did not receive any specific grant from any funding agency in the public, commercial or not-for-profit sector.

\section{Acknowledgements}

The authors wish to thank Dr Giulia Di Dalmazi (Department of Pathology, The Johns Hopkins School of Medicine, Baltimore) for pituitary antibodies assay, Dr Francesco Belotti (Unit of Otorhinolaryngology, University of Brescia, Brescia, Italy) for drawing Fig. 1, and Stephanie Marino for critical revision of the manuscript.

\section{References}

1 Honegger J, Schlaffer S, Menzel C, Droste M, Werner S, Elbelt U, Strasburger C, Stormann S, Kuppers A, Streetz-van der Werf C et al. Diagnosis of primary hypophysitis in Germany. Journal of Clinical Endocrinology and Metabolism 2015100 3841-3849. (doi:10.1210/ jc.2015-2152)
2 Caturegli P, Newschaffer C, Olivi A, Pomper MG, Burger PC \& Rose NR. Autoimmune hypophysitis. Endocrine Reviews 200526 599-614. (doi:10.1210/er.2004-0011)

3 Leporati P, Landek-Salgado MA, Lupi I, Chiovato L \& Caturegli P. IgG4-related hypophysitis: a new addition to the hypophysitis spectrum. Journal of Clinical Endocrinology and Metabolism 201196 1971-1980. (doi:10.1210/jc.2010-2970)

4 Lupi I, Manetti L, Raffaelli V, Lombardi M, Cosottini M, Iannelli A, Basolo F, Proietti A, Bogazzi F, Caturegli P et al. Diagnosis and treatment of autoimmune hypophysitis: a short review. Journal of Endocrinological Investigation 201134 e245-e252. (doi:10.3275/7863)

5 Honegger J, Buchfelder M, Schlaffer S, Droste M, Werner S, Strasburger C, Stormann S, Schopohl J, Kacheva S, Deutschbein $\mathrm{T}$ et al. Treatment of primary hypophysitis in Germany. Journal of Clinical Endocrinology and Metabolism 2015100 3460-3469. (doi:10.1210/jc.2015-2146)

6 Turcu AF, Erickson BJ, Lin E, Guadalix S, Schwartz K, Scheithauer BW, Atkinson JL \& Young WF Jr. Pituitary stalk lesions: the Mayo Clinic experience. Journal of Clinical Endocrinology and Metabolism 201398 1812-1818. (doi:10.1210/jc.2012-4171)

7 Bogazzi F, Manetti L, Lombardi M, Giovannetti C, Raffaelli V, Urbani C, Scattina I, Pepe P, Iannelli A, Martino E et al. Impact of different cut-off limits of peak GH after GHRH-arginine stimulatory test, single IGF1 measurement, or their combination in identifying adult patients with GH deficiency. European Journal of Endocrinology 2011164 685-693. (doi:10.1530/EJE-10-1068)

8 Corneli G, Di Somma C, Baldelli R, Rovere S, Gasco V, Croce CG, Grottoli S, Maccario M, Colao A, Lombardi G et al. The cut-off limits of the $\mathrm{GH}$ response to GH-releasing hormone-arginine test related to body mass index. European Journal of Endocrinology 2005153 257-264. (doi:10.1530/eje.1.01967)

9 Fleseriu M, Hashim IA, Karavitaki N, Melmed S, Murad MH, Salvatori R \& Samuels MH. Hormonal replacement in hypopituitarism in adults: an Endocrine Society Clinical Practice Guideline. Journal of Clinical Endocrinology and Metabolism 2016101 3888-3921. (doi:10.1210/jc.2016-2118)

10 Ricciuti A, De Remigis A, Landek-Salgado MA, De Vincentiis L, Guaraldi F, Lupi I, Iwama S, Wand GS, Salvatori R \& Caturegli P. Detection of pituitary antibodies by immunofluorescence: approach and results in patients with pituitary diseases. Journal of Clinical Endocrinology and Metabolism 201499 1758-1766. (doi:10.1210/ jc.2014-1049)

11 Simmons GE, Suchnicki JE, Rak KM \& Damiano TR. MR imaging of the pituitary stalk: size, shape, and enhancement pattern. American Journal of Roentgenology 1992159 375-377. (doi:10.2214/ ajr.159.2.1632360)

12 Grossman JM, Gordon R, Ranganath VK, Deal C, Caplan L, Chen W, Curtis JR, Furst DE, McMahon M, Patkar NM et al. American College of Rheumatology 2010 recommendations for the prevention and treatment of glucocorticoid-induced osteoporosis. Arthritis Care and Research 201062 1515-1526. (doi:10.1002/acr.20295)

13 Nakata Y, Sato N, Masumoto T, Mori H, Akai H, Nobusawa H, Adachi Y, Oba H \& Ohtomo K. Parasellar T2 dark sign on MR imaging in patients with lymphocytic hypophysitis. American Journal of Neuroradiology 201031 1944-1950. (doi:10.3174/ajnr.a2201)

14 Gutenberg A, Larsen J, Lupi I, Rohde V \& Caturegli P. A radiologic score to distinguish autoimmune hypophysitis from nonsecreting pituitary adenoma preoperatively. American Journal of Neuroradiology 200930 1766-1772. (doi:10.3174/ajnr.a1714)

15 Sbardella E, Joseph RN, Jafar-Mohammadi B, Isidori AM, Cudlip S \& Grossman AB. Pituitary stalk thickening: the role of an innovative MRI imaging analysis which may assist in determining clinical management. European Journal of Endocrinology 2016175 255-263. (doi:10.1530/EJE-16-0455) 
16 Takao T, Nanamiya W, Matsumoto R, Asaba K, Okabayashi T $\&$ Hashimoto K. Antipituitary antibodies in patients with lymphocytic hypophysitis. Hormone Research 200155 288-292. (doi:10.1159/000050015)

17 Lupi I, Broman KW, Tzou SC, Gutenberg A, Martino E \& Caturegli P. Novel autoantigens in autoimmune hypophysitis. Clinical Endocrinology 200869 269-278. (doi:10.1111/j.1365-2265.2008.03180.x)

18 De Bellis A, Pane E, Bellastella G, Sinisi AA, Colella C, Giordano R, Giavoli C, Lania A, Ambrosio MR, Di Somma C et al. Detection of antipituitary and antihypothalamus antibodies to investigate the role of pituitary or hypothalamic autoimmunity in patients with selective idiopathic hypopituitarism. Clinical Endocrinology 201175 361-366. (doi:10.1111/j.1365-2265.2011.04056.x)

19 Tzou SC, Lupi I, Landek M, Gutenberg A, Tzou YM, Kimura H, Pinna G, Rose NR \& Caturegli P. Autoimmune hypophysitis of SJL mice: clinical insights from a new animal model. Endocrinology 2008 149 3461-3469. (doi:10.1210/en.2007-1692)

20 Byrne TN, Stone JH, Pillai SS, Rapalino O \& Deshpande V.

Case records of the Massachusetts General Hospital. Case 31-2016. A 53-year-old man with diplopia, Polydipsia, and Polyuria. New England Journal of Medicine 2016375 1469-1480. (doi:10.1056/ NEJMcpc1610097)

Received 12 February 2017

Revised version received 19 April 2017

Accepted 11 May 2017 